\title{
Representing Layout Information in the CMSD Specification
}

\author{
Frank Riddick \\ Y. Tina Lee \\ Manufacturing Systems Integration Division \\ National Institute of Standards and Technology \\ 100 Bureau Drive \\ Gaithersburg, MD 20899, USA \\ 301-975-3892, 301-975-3550 \\ riddick@nist.gov, leet@nist.gov
}

Keywords:

CMSD, data exchange, information model, and manufacturing simulation

\begin{abstract}
ABSTRCT: Developing mechanisms for the efficient exchange of information between simulations and other manufacturing tools is a critical problem. For many areas of manufacturing, neither representations for the information nor mechanisms for exchanging the information have been agreed upon. Manufacturing plant layout is one such area. The Core Manufacturing Simulation Data (CMSD) specification is being developed to address some of these issues, through the definition of neutral representations for the "core" manufacturing entities that need to be exchanged between simulations and other applications, through the creation of a Unified Modeling Language information model that defines the relationships between the core manufacturing entities, and through the definition of eXtensible Modeling Language Schemas based on the information model to facilitate the exchange of information that adheres to the model. This paper describes an effort to extend the CMSD specification to cover the definition and exchange of layout information.
\end{abstract}

\section{Introduction}

Simulation technology has been demonstrated to be an effective tool for reducing costs, improving quality, and shortening the time-to-market for manufactured goods. However, there are a number of technical and economic barriers that hinder the use of this technology in the manufacturing industry. The cost of developing, implementing, and using simulation technology is high. The costs of integrating simulation systems with other manufacturing applications are even higher. There is always a need to transfer, exchange, and share data between simulation and other manufacturing applications. Custom-built, proprietary interfaces are costly and make it prohibitive expensive for users to exploit simulation technology. The development of reusable, neutral, standard interfaces would make it easier and could help reduce the costs to integrate simulation and other manufacturing applications. National Institute of Standards and Technology (NIST) researchers, in collaboration with industrial partners, have been involved in developing a standard called the Core Manufacturing Simulation Data (CMSD) specification under the guidelines, policies, and procedures of the Simulation Interoperability Standards Organization (SISO).
The CMSD specification provides a means to define information about many kinds of manufacturing entities such as resources, parts, and process plans. One area of information that was not covered in previous versions of CMSD is the area of plant layout. "Layout refers to the efforts involved in selecting specific locations for each department, process, machine, support functions, and other activity that will be a part of the operations at a facility" [1]. There are many potential simulation applications that might make use of layout information, such as simulations looking at ergonomic issues, material handling issues, or comparisons of the effects of different layouts on production operations. Simulation can provide an effective means to evaluate many different alternatives involving layout issues, without incurring the cost and effort of physically modifying existing facilities. As with other manufacturing areas, there are no widely accepted standards for exchanging layout information. Extending CMSD to support the definition and exchange of layout information will help address this issue.

In the following sections, information about how the CMSD specification was extended to support layout information is presented. Section 2 presents a brief overview of CMSD, its goals, and the manufacturing areas covered. In section 3, goals and requirements for the top 
level structures added to CMSD to support layout information are discussed, and Unified Modeling Language (UML) class diagrams depicting the layout structures is presented. In section 4, activities that have been undertaken to evaluate the effectiveness of the CMSD layout extensions will be discussed. The paper concludes with section 5 where a summary of the paper is presented.

\section{CMSD Overview}

Information management problems affect many aspects of manufacturing operations, but they are a particular hindrance to the creation and reuse of manufacturing simulations. The CMSD specification addresses issues related to information management and manufacturing simulation development. It provides a neutral framework that facilitates the creation of collections of related manufacturing information suitable for use in the creation or enhancement of manufacturing simulations and other manufacturing applications. The entities defined in this framework represent a core set of the manufacturing entities and relationships needed for manufacturing simulation.

\subsection{CMSD major information categories}

Representations for many kinds or categories of manufacturing information are present in the CMSD specification. Below is a description of some of the major categories of CMSD information.

- Calendar information describes the groups of shifts (and their related break and holiday information) that are in effect for a specified time period.

- Resource information describes the people and equipment that perform manufacturing activities. Resources in CMSD are used to represent stations, machines, cranes, employees, tools, and fixtures.

- Skill information describes the skills that an employee resource may possess and the levels of proficiency associated with those skills.

- Setup information describes how resources may be configured to perform a task, how long it takes to configure the resource, and how long it takes to change from one configuration to another.

- Part information provides a means to specify the characteristics of the materials and subcomponents that are used to make end products.

- Bill-of-materials information provides a means to specify the subcomponent parts and the quantities of those parts that are needed to make an end product.

- Inventory information provides a means to specify the location and characteristics of instances of the parts, materials, tools, and fixtures used in production.

- Process plan information specifies the set of production activities needed to transform materials and subcomponents into finished products.

- Maintenance plan information defines preventive or corrective maintenance actions that are to be done on machines or other maintained resources.

- Order and Job information provides a means to create descriptions of the production activities that will be used to produce a product, along with estimates of the effort necessary to carry out those activities.

- Schedule information provides a means to define lists of production activities that are planned to occur during a specified time period.

- Reference information provides a means to associate the entities defined in the CMSD with information defined outside of the CMSD, facilitating the assembly of rich collections of associated information from diverse sources.

- Probability distribution information provides a means to specify statistical distributions that can be used to indicate process variability.

\subsection{The use of multiple modeling languages in defining CMSD}

Taken together, the data definitions and interrelationships defined in the CMSD specification create an information model intended to facilitate the exchange of data between simulations and other manufacturing applications. Several modeling languages are used to define the CMSD information model. Class and package diagrams using UML have been defined to provide a visual definition of the model. In addition, a series of eXtensible Modeling Language (XML) Schemas have been defined based on the UML version of the CMSD information model. The exchange of data between simulations will be enabled through the exchange of XML instance documents that adhere to the CMSD XML Schemas. Also, where necessary, the CMSD XML schemas have been enhanced with embedded Schematron information. Schematron, an ISO standard, is a rules-based language for specifying constraints on information in XML documents. Since there are some constraints defined in the CMSD UML model that cannot be expressed using the XML Schema 
language, Schematron was used to specify those constraints.

The UML diagrams and the XML schemas are intended to be identical representations of the same CMSD conceptual model. The UML representation provides a means to express the complex interrelationships inherent in the manufacturing information using a visual medium and format that is commonly used in the technical community. The XML schema representation provides a machine interpretable form of the model that can be used to validate exchanged information using easily available tools that can process XML data.

\section{Extending the CMSD Specification to Support Layout}

As stated above, the CMSD specification was created to facilitate the exchange of information between simulations and other manufacturing applications covering a number of different manufacturing domains. Since many simulation applications have a need to provide basic visualizations of the manufacturing entities and facility operations being simulated, extending CMSD to support layout information should expand the potential number of applications in which CMSD-based information exchange can be used. Following is a discussion of the goals and requirements that the CMSD layout extensions are intended to address, and a description of the top level structures developed to meet those goals and requirements.

\subsection{Goals for the CMSD layout extensions}

In CMSD, layout information is used to define spatiallyoriented characteristics and interrelationships for the logical and physical entities that are used to carry out production activities. The information in a layout is intended to facilitate the visual analysis of those entities' spatial relationships and characteristics in a simulated manufacturing environment. In addition, depending on the precision with which the characteristics and interrelationships of the entities have been specified, the creation of non-visually-oriented analysis applications is also facilitated. Finally, as a part of the CMSD specification, the ability to associate spatially-oriented data (defined based on CMSD's layout structures) with nonspatially-oriented data (defined based on structures from the resource, part, process planning, and other parts of the CMSD specification) is required.

An additional goal for the CMSD specification's support for the definition of layout information is that the definitions be neutral. The CMSD specification is not based or dependent on proprietary specifications or other intellectual property, and is not skewed to better support any existing commercial off the shelf (COTS) product over another.

\subsection{CMSD's relationship to existing approaches for modeling layout information}

As a part of the effort to develop an approach for the specification of layout data related to simulation, the Simulation Data eXchange specification (SDX) was examined [2]. SDX is an effort by UGS (now Siemens PLM Software) to develop a specification that can be used to exchange data between simulation systems and other manufacturing applications. It provides a means to represent the layout and related spatial characteristics of the machines used in a manufacturing facility to produce a product. Some process related and simulation execution related information can also be specified. While there are many useful aspects of the SDX format, its proprietary nature does not allow CMSD's layout modeling capabilities to be directly based upon it.

Another common approach today is to use ComputerAided-Design (CAD) formats to model, store, and exchange layout information. These formats provide a means to rigorously specify, with a high degree of precision and fidelity, geometric information about the entities in a manufacturing facility, such that the specified information can be interpreted as a near-realistic-virtual representation of those manufacturing entities. While this strategy may be suitable for some manufacturing analysis applications, there are a couple of problems inherent with employing it.

There are a myriad of CAD formats and technologies to translate between those formats. Interoperability between these formats is a well documented problem [3]. Also, many of these formats are proprietary. To address the interoperability and intellectual property issues, several existing standardization efforts have been undertaken, including the STandard for the Exchange of Product model data (STEP) [4] and the Initial Graphics Exchange Specification (IGES) [5].

The layout portion of the CMSD specification is NOT intended to be a new CAD format. As stated above, there are already existing efforts underway to address CAD format issues. Also, even though many applications need to be able to deal with some of the geometric or spatial aspects of manufacturing entities, the level to which these applications need to process that information can differ greatly. 
Some analysis applications, such as Finite Element Analysis applications, require the precise definitions for the geometry of the entities being modeled that CAD systems can provide. On the other hand, many simulation applications only need a general definition of the boundaries, placement, and some other spatially-related aspects of the manufacturing entities being simulated, and a way to associate that information with non-spatiallyrelated information about the entities. These applications are focused primarily on analyzing, evaluating, and optimizing the processes that take place as part of production operations. While some spatially-oriented information may be needed, highly detailed, high fidelity geometric representations of the manufacturing entities being modeled are not required. Applications which have these basic spatial information requirements are the types of applications for which the layout portion of the CMSD specification is intended to be useful.

\subsection{Requirements for the CMSD specification's top- level layout structures}

The layout structures in CMSD are intended to provide a means to specify the following:

- The coordinate system and spatial dimensions defining the boundary for a conceptual or existing area/volume within which production operations take place.

- The coordinate system and spatial dimensions defining the boundary for conceptual or real manufacturing entities that are involved in production operations.

- Placement and transformation information defining the spatial relationships between the manufacturing entities defined in a layout and their enclosing layout.

- Definitions for and placement and transformation information for graphical representations of manufacturing entities in a layout.

- Definitions for and placement and transformation information for textual annotations that are to be depicted when a layout is visualized.

- Information associating a layout and/or the manufacturing entities contained in a layout with resource- and/or process- related manufacturing information defined by other CMSD elements.

In the next section, a UML model depicting the top-level structures added to the CMSD specification will be presented. This is only a partial representation of all of the structures added to the CMSD specification to support layout information. The team developing the CMSD specification intends to publish information about the other structures and the requirements these structures are intended to support in the future.

\subsection{Top-level CMSD UML layout class overview}

In general, the CMSD layout specification provides a means to define the boundaries of a "space" in which production activities may take place and to define the boundaries, orientation, and, optionally, a basic visual representation of the "objects" within that space that are involved in carrying out those production activities. Below is a brief description about the top level CMSD classes that are used to describe layout information.

Figure 1 and figure 2 present UML class diagrams that show the definitions for the main classes added to the CMSD specification to support the definition of layout information. The Layout class provides the means to specify information about the space within which production takes place, and the LayoutObject class provides the means to specify information about the type and shape of objects within that space.

Both the Layout class and LayoutObject class are children of the abstract LayoutElement class. From this class they inherit the ability to define boundary information (using the BoundaryDefinition class) and to be associated with manufacturing resource information (non-spatially related information about machines, conveyors, stations, etc.,) defined by other classes in the CMSD specification. The Placement class provides the means for each Layout instance to define information about the location, orientation, and scaling of LayoutElement instances that spatially exist within the boundaries of the (enclosing) Layout instance.

Layout and LayoutObject instances can be used to define the spatially-oriented information about a factory layout. This information generally corresponds to the manufacturing object types defined by SDX, and can be used by layout-analysis applications as a suggestion for how the spatial information should be interpreted, or to facilitate the use of different visualizations for the different kinds of layout information.

A complete set of UML classes, diagrams, and matching XML schema definitions have been created for the layout extensions to CMSD, but due to space concerns only the classes and their associated descriptions that are essential for understanding layout definition are presented here. To verify that the extended CMSD specification can be used to exchange layout information, several verification activities were performed. In the following section, a description of these activities is presented. 


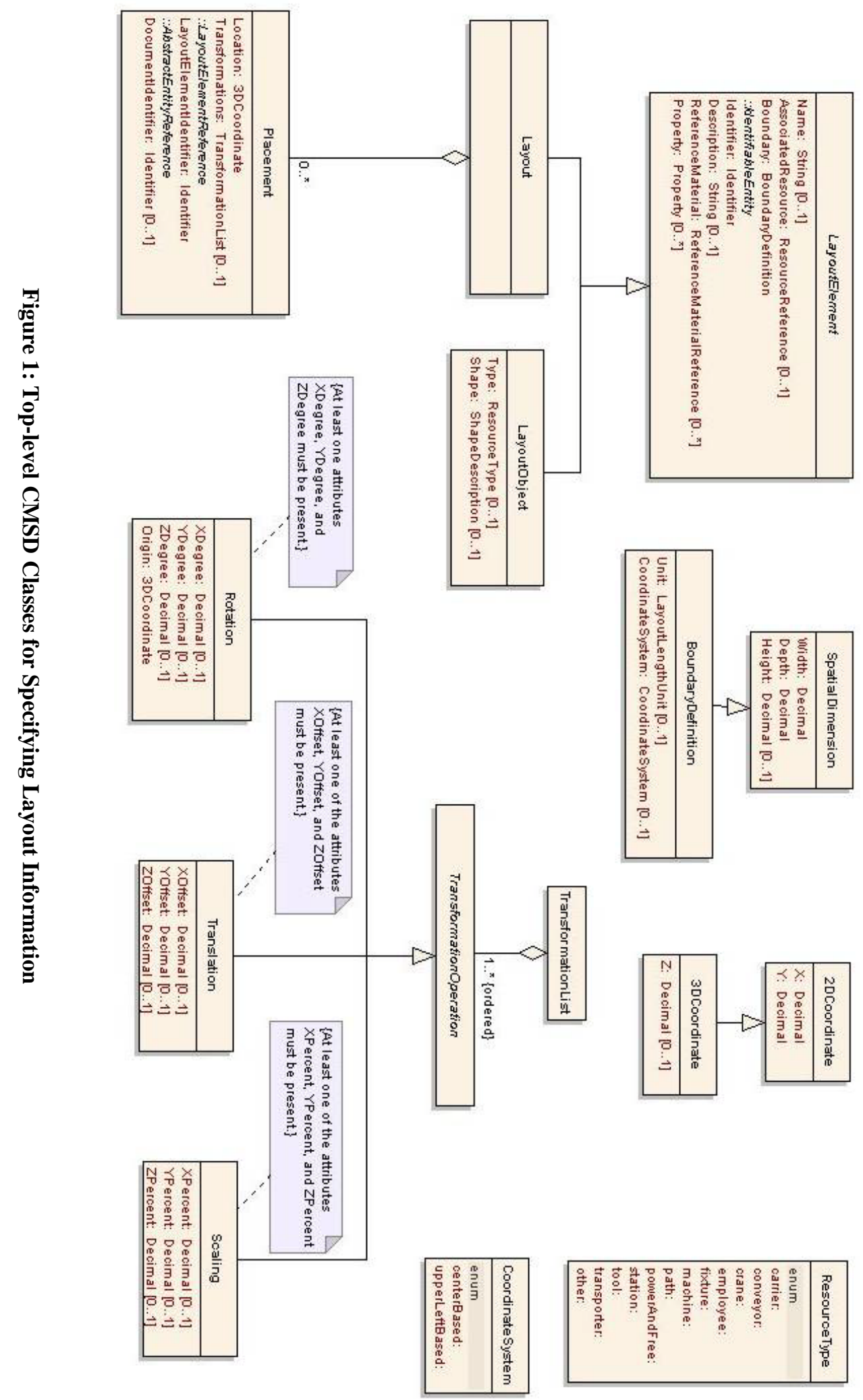




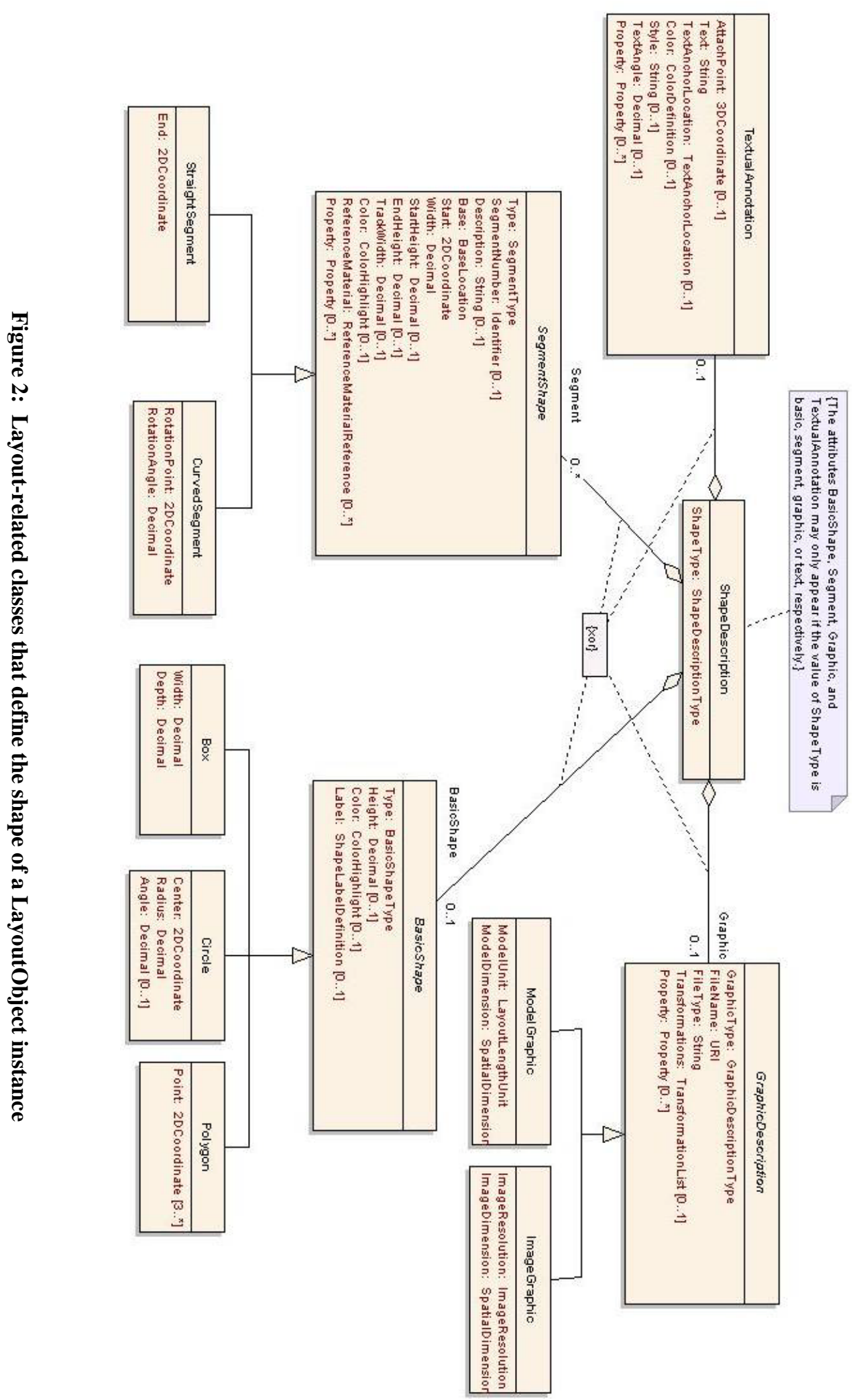




\subsection{An SVG-based 2D Layout viewing application}

\section{CMSD Layout Extensions Verification Activities}

As a part of an effort to verify that information exchange based on the CMSD specification is a viable approach, several projects involving CMSD and the integration of real manufacturing systems were undertaken. These projects allow the CMSD development team and its collaborators to assess the feasibility of using CMSD to integrate manufacturing application and simulations and to uncover the potential for new modifications and/or extensions. Information about some of these projects can be found in [6, 7, and 8]. As a first step in evaluating the feasibility of the layout extensions to CMSD, several activities to evaluate CMSD's ability to represent the information of a basic factory layout were undertaken. The objective was to create an example file (formatted according to the CMSD specification) containing information about a simple facility layout and to create simple viewing applications for the information in the file using different viewing technologies. Information about the results of those activities is presented below.

\subsection{Representing Layout information in CMSD}

Many organizations have published on the internet information about and images of their facility layout. Several example layout images were collected, and an image of a basic facility layout was chosen as the first subject of the activity. The image is depicted in Figure 3.

The pixels in the image were analyzed manually to determine the boundaries and relative placement of the different areas. Based on the information generated from the manual inspection of the image, an XML instance document adhering to the CMSD specification was created. The file was validated against the CMSD XML schemas that have been extended to cover layout information.

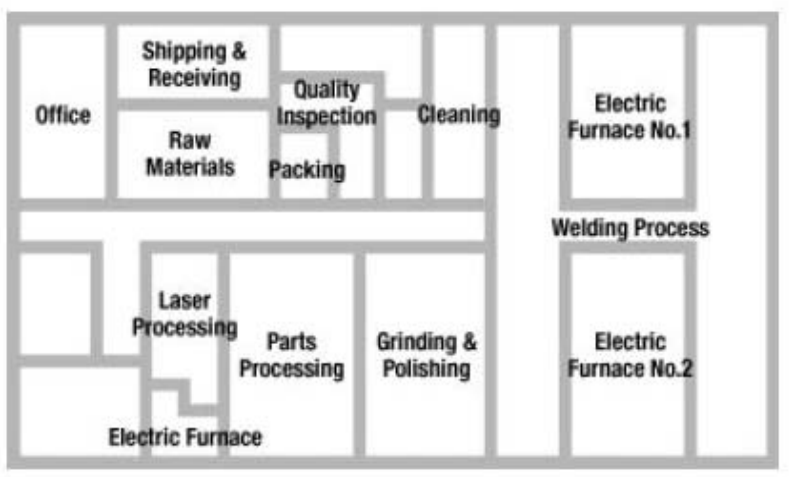

Figure 3: Example Factory Layout
Since the example layout information was contained in an XML file, the first attempt to create a viewing application looked at using only basic technologies approved by the World Wide Web Consortium (W3C). An eXtensible Stylesheet Language (XSL) file was created that transformed the CMSD XML file into an XML file adhering to the Scalable Vector Graphic (SVG) specification [9]. SVG is a W3C approved specification that supports the display of 2D graphics on the web. Many browsers directly support the display of SVG-based information. Figure 4 is an image generated from the SVG file.

This application is intended only to show that the information in the test CMSD file can be used to create a basic depiction of the boundaries and relative positions of the layout objects in the layout. The names of the layout objects are contained in the CMSD XML file, but they are not displayed due to limitations in the text processing capabilities in SVG. A more complex SVG conversion and display process is possible but would involve the use and integration of other technologies in the process, and as such was considered beyond the scope of the intended simple viewing application.

Note that in a couple of areas, there appear to be some extraneous lines. These are not errors, but are instead indications of overlap between some of the layout objects. In the CMSD extensions for layout, overlap between objects is not considered an error. It is the responsibility of the viewing or other analysis application to determine if overlap between layout objects is allowed, and to take appropriate action if necessary.

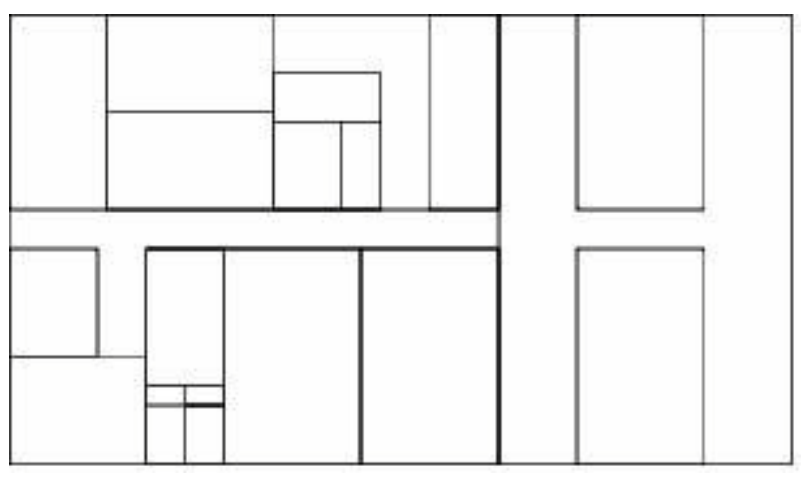

Figure 4: 2D Layout Rendered using SVG

\subsection{A Flex-based 2D Layout viewing application}


To determine if some of the limitations of the SVG-based viewing application could be overcome, a different viewing application was created. This application was developed using the Actionscript language, Flex framework, and Flash player application created by Adobe [10]. Flex-based technologies are widely used by developers creating rich internet applications, and Adobe recently released these technologies as open source. These technologies provide a number of different options for interpreting and displaying CMSD-based information. The Flex-based application uses the same CMSD-based XML file that was used by a previous application to generate a $2 \mathrm{D}$ visualization. In addition to supporting the placement of LayoutObjects within the Layout, the automatic placement of descriptive information specified in the XML file is also supported by this application. Figure 5 shows the image generated from the application.

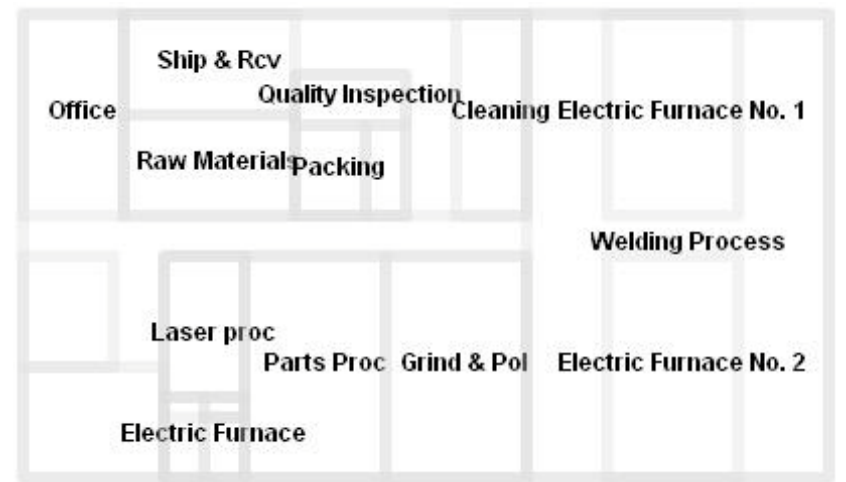

Figure 5 - 2D Layout Rendered using Flex

\subsection{Flex-based 3D layout viewing application}

In the effort to extend the CMSD specification to support the definition of layout information, the initial focus has been to ensure that, at a minimum, 2D layouts can be created from information adhering to the specification. Many simulation packages and related manufacturing tools only have support for the definition, manipulation, and analysis of spatial data in 2 dimensions, but there are many others that support or require data defined with 3 dimensions. In order to be able to support both kinds of applications, structures that define points or spatial boundaries related to Layout or LayoutObject instances may be defined using either 2 or 3 dimensions.

To determine the feasibility of this approach, a Flexbased application that displays a 3D layout representation using XML data adhering to the CMSD specification was developed. To support this application, the CMSD XML document used with the first two applications is enhanced so that all the data is 3 dimensional. This new CMSD file is read into a Flex-based application that can display information in 3D through the use of an open source enhancement to the Flex framework. Figure 6 shows an image generated from the current version of the application. This application is still under development, but initial results indicated that the layout enhancements to CMSD specification are able to support the definition of both 2D and 3D layout information.

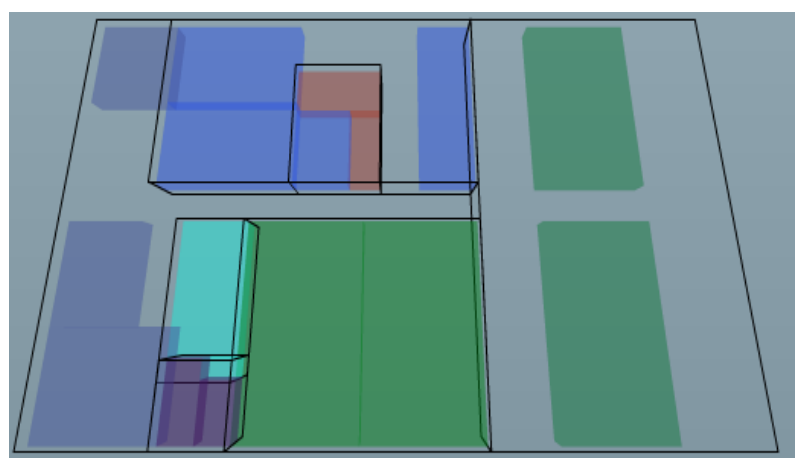

Figure 6: 3D Layout Rendered using Flex

\section{Summary}

In this paper, information about enhancements to the CMSD specification to support the definition and exchange of layout information was presented. The overall goals for the layout extensions, the requirements that need to be met, and the relevance of related standardization efforts were discussed. A UML diagram depicting the top level structures that were added to the CMSD specification was presented. Finally, information about verification efforts involving several layout display applications that used CMSD formatted layout information as input was presented.

\section{ACKNOWLEDGMENT}

Work described in this report was sponsored by the National Institute of Standards and Technology (NIST) Simulation-based Manufacturing Interoperability Standards and Testing Program, Gaithersburg, Maryland. The program supports NIST projects applying information technologies and standards-based approaches to manufacturing software integration problems. The work described was funded by the United States Government and is not subject to copyright.

\section{DISCLAIMER}

A number of software products are identified in context in this paper. This does not imply a recommendation or endorsement of the software products by the authors or NIST, nor does it imply that such software products are necessarily the best available for the purpose. 


\section{REFERENCES}

[1] Siegel, J., and J. K. Shim. 1999. Operations Management, $1^{\text {st }}$ ed. Hauppauge, New York: Barron's Educational Series, Inc.

[2] Moorthy, S. 1999. Integrating the CAD Model With Dynamic Simulation: Simulation Data Exchange, In Proceedings of the 1999 Winter Simulation Conference, 276-280. New York: Association for Computing Machinery.

[3] Biaconi, F., P. Conti, and L. Angelo. 2006. Interoperability among CAD/CAM/CAE Systems: A Review of Current Research Trends. In Proceedings of the Geometric Modeling and Imaging -- New Trends Conference, 82-89. London: IEEE Computer Society.

[4] ISO 10303-1. 1994. Part 1: Overview and Fundamental Principles. Industrial Automation Systems and Integration-Product Data Representation and Exchange. Geneva, Switzerland: International Organization for Standardization.

[5] U. S. Product Data Association. 1996. Initial Graphics Exchange Specification IGES 5.3. Charleston, South Carolina: U. S. Product Data Association.

[6] Johansson, M., S. Leong, Y. T. Lee, F. Riddick, G. Shao, B. Johansson, A. Skoogh, and P. Klingstam. 2007. A Test Implementation of the Core Manufacturing Simulation Data Specification, In Proceedings of the 2007 Winter Simulation Conference, 1673-1681. Piscataway, New Jersey: Institute of Electrical and Electronics Engineers, Inc.

[7] Kibira, D., and C. McLean. 2007. Generic Simulation of Automotive Assembly for Interoperability Testing. In Proceedings of the 2007 Winter Simulation Conference, 1035-1043. Piscataway, New Jersey: Institute of Electrical and Electronics Engineers, Inc.

[8] Lee, Y. T., S. Leong, F. Riddick, M. Johansson, and B. Johansson. 2007. A Pilot Implementation of the Core Manufacturing Simulation Data Information Model. In Proceedings of the Simulation Interoperability Standards Organization 2007 Fall Simulation Interoperability Workshop. Orlando, Florida: Simulation Interoperability Standards Organization, Inc.

[9] Ferraiolo, J., J. Fujisawam, and D. Jackson, editors. 2003. Scalable Vector Graphics (SVG) 1.1 Specification. World Wide Web Consortium. Available via <http://www.w3.org/TR/SVG11/> [accessed on June 13, 2008].

[10]Lott, J., D. Schall, and K. Peters. 2006. ActionScript 3.0 Cookbook: Solutions for Flash Platform and Flex Application Developers. Sebastopol, California: O’Reilly Media, Inc.

\section{AUTHOR BIOGRAPHIES}

FRANK RIDDICK is a computer scientist in the Manufacturing Simulation and Modeling Group in The
National Institute of Standards and Technology (NIST) Manufacturing Systems Integration Division. He has participated in research and authored several papers relating to manufacturing simulation integration and product data modeling. He holds a Master's Degree in Mathematics from Purdue. His email address is <frank.riddick@nist.gov>.

Y. TINA LEE is a computer scientist in the Manufacturing Simulation and Modeling Group at NIST. She joined NIST in 1986. Her major responsibility in recent years has been to develop information models to support various manufacturing application areas. Previously she worked at the Contel Federal Systems and at the Sperry Corporation. She received her BS in Mathematics from Providence College and MS in Applied Science from the College of William and Mary. Her e-mail address is<leet@nist.gov>. 